\title{
On the Stability of Distance-based Formation Control
}

\author{
Dimos V. Dimarogonas and Karl H. Johansson
}

\begin{abstract}
This paper examines stability properties of distance-based formations. These are formations encoded by inter-agent relative distances. A negative gradient control law is proposed and is shown to be provably correct when the formation graph is a tree. Moreover, it is shown that the tree structure is a necessary and sufficient condition for distancebased formation stabilization with negative gradient control laws. For graphs that contain cycles, a characterization of the resulting equilibria is given based on the properties of the cycle space of the graph. The results are also applied to flocking motion for double integrator agents.
\end{abstract}

\section{INTRODUCTION}

Decentralized control of networked multi-agent systems is a field of increasing research interest, due to its applications in multi-robot systems [14], air traffic management systems [24] and distributed control of multiple vehicles [21]. A particular problem considered in literature is that of multi-agent formation control, where agents usually represent multiple robots or vehicles that aim to converge to a specified pattern in the state space. The desired formation can be either static [23],[3],[5] or moving with constant velocity [18],[22].

Among the vast literature on formation control, two main approaches can be distinguished: position-based and distance-based formation control. In the first case, agents aim to converge to desired relative position vectors with respect to a subset of the rest of the team. Control designs that guarantee position-based formation stabilization have appeared for single integrator agents [23],[5],[13] as well as nonholonomic agents [3],[16]. Although position-based formation stabilization is a well studied topic, there appears to be a lack of relevant results for the case of distancebased formations. Such formation are useful in some cases where limited knowledge of global coordinates prevents the agents from having defined desired relative orientations. The purpose of this paper is to examine particular properties of control schemes for such formations.

Distance-based formations have been studied in the context of graph rigidity where a series of results have appeared in recent literature, e.g., [1], [19],[7], [11]. Roughly speaking, a formation is called rigid if the fact that all desired distances are met is sufficient for the maintenance of the distances of any pair of agents. Necessary and sufficient conditions for graph rigidity have been provided in [6], [11]; refer to the

The authors are with KTH ACCESS Linnaeus Center, School of Electrical Engineering, Royal Institute of Technology (KTH), Stockholm, Sweden dimos, kallejdee.kth.se. This work was done within TAISAURES program (297316-LB704859), funded by the Swedish Governmental Agency for Innovation Systems (VINNOVA) and the Swedish Defence Materiel Administration (FMV). It was also supported by the Swedish Research Council, the Swedish Foundation for Strategic Research, and the EU NoE HYCON. recent $\mathrm{PhD}$ thesis [10] and the references therein for more information on graph rigidity. A common factor in the graph rigidity literature is the lack of stabilizing control laws that drive the agents to the desired distance. Existing control laws such as the one proposed in [1] only have local validity for small perturbations around the desired formation.

Motivated by the lack of control laws and stability analysis, we pursue in this paper the problem of distance-based formation control. In particular, we propose a control law that is based on the negative gradient of a potential function between each of the pairs of agents that form an edge in the formation graph. In the first result, we design such a function and show that it stabilizes the system to the desired relative distances provided that the formation graph is a tree. It is then shown that a tree is also a necessary condition for stabilization to the desired formation. Thus, another contribution of the paper is that it is proven that control laws consisting of negative gradients of potential fields can only stabilize the system when the graph is a tree, whereas no global stabilization guarantees for graphs that contain cycles can be provided. In essence, rigid formations are not globally stabilizable with negative gradient control laws. What is shown though is that for some graphs with cycles, a characterization of the resulting equilibria can be given, based on the properties of the cycle space of the graph.

The rest of the paper is organized as follows: in Section II the problem treated is presented. Section III includes the graph theoretic tools used in the paper. The control law and stability analysis are presented in Section IV. Section V extends the results to the case of flocking motion for double integrator agents. Computer simulations are included in Section VI and the results are summarized in Section VII.

\section{System And PRoblem Statement}

Consider a system of $N$ kinematic agents operating in $\mathbb{R}^{2}$. Let $q_{i} \in \mathbb{R}^{2}$ denote the position of agent $i$. The configuration space is spanned by $q=\left[q_{1}^{T}, \ldots, q_{N}^{T}\right]^{T}$. We assume first that agents' motion obeys the single integrator model:

$$
\dot{q}_{i}=u_{i}, i \in \mathcal{N}=\{1, \ldots, N\}
$$

where $u_{i}$ denotes the velocity (control input) for each agent.

The objective of the control design is distance-based formation control. As mentioned in the introduction, the innovation of the paper is that it provides stability guarantees for this type of formations. While numerous results have appeared in literature involving agents that aim to converge to desired relative positions, there seem to be no results for the case when the rotation objective is omitted, i.e., when agents aim to converge to desired relative distances, instead 
of relative positions. We provide in the sequel a control law and a sufficient condition on the network topology that guarantee such formation stabilization.

Each agent can only communicate with a specific subset $N_{i} \subset \mathcal{N}$. By convention, $i \notin N_{i}$. The desired formation can be encoded in terms of an undirected graph, from now on called the formation graph $G=\{\mathcal{N}, E\}$ [17], whose set of vertices $\mathcal{N}=\{1, \ldots, N\}$ is indexed by the team members, and whose set of edges $E=\left\{(i, j) \in \mathcal{N} \times \mathcal{N} \mid j \in N_{i}\right\}$ contains pairs of vertices that represent inter-agent formation specifications. Each edge $(i, j) \in E$ is assigned a scalar parameter $d_{i j}=d_{j i}>0$, representing the distance at which agents $i, j$ should converge to. Define the set

$$
\Phi \triangleq\left\{q \in \mathbb{R}^{2 N} \mid\left\|q_{i}-q_{j}\right\|=d_{i j}, \forall(i, j) \in E\right\}
$$

of desired distance based formations. The desired formation is called feasible if $\Phi$ is non-empty. The problem treated in the first part of the paper is summarized as follows: derive control laws, for which the information available for each agent $i$ is encoded in $N_{i}$, that drive the single integrator agents to the desired formation, i.e., $\lim _{t \rightarrow 0} q(t)=q^{*} \in \Phi$.

In the last part, we apply the results to a distance based flocking problem. We assume that agents obey now a double integrator model of the form:

$$
\begin{aligned}
& \dot{q}_{i}=u_{i} \\
& \dot{u}_{i}=v_{i}
\end{aligned}, i \in \mathcal{N}
$$

The objective of the control design is now the movement of all agents with a common velocity while achieving at the same time the desired distance based formation.

\section{ELEMENTS FROM GRAPH THEORY}

We first review in this section some elements of algebraic graph theory [8] used in the sequel and also present a lemma that is important for the subsequent analysis.

For an undirected graph $G$ with $N$ vertices the adjacency matrix $A=A(G)=\left(a_{i j}\right)$ is the $N \times N$ matrix given by $a_{i j}=1$, if $(i, j) \in E$ and $a_{i j}=0$, otherwise. If there is an edge $(i, j) \in E$, then $i, j$ are called adjacent. A path of length $r$ from a vertex $i$ to a vertex $j$ is a sequence of $r+1$ distinct vertices starting with $i$ and ending with $j$ such that consecutive vertices are adjacent. For $i=j$, this path is called a cycle. If there is a path between any two vertices of the graph $G$, then $G$ is called connected (otherwise it is called disconnected). A connected graph is called a tree if it contains no cycles. The degree $d_{i}$ of vertex $i$ is defined as the number of its neighboring vertices, i.e. $d_{i}=\left|N_{i}\right|$. Let $\Delta$ be the $n \times n$ diagonal matrix of $d_{i}$ 's. The (combinatorial) Laplacian of $G$ is the symmetric positive semidefinite matrix $L=\Delta-A$. For a connected graph, the Laplacian has a single zero eigenvalue and the corresponding eigenvector is the vector of ones, $\overrightarrow{\mathbf{1}}$.

An orientation on the graph $G$ is the assignment of a direction to each edge. The graph $G$ is called oriented if it is equipped with a particular orientation. The incidence matrix $B=B(G)=\left(B_{i j}\right)$ of an oriented graph is the $\{0, \pm 1\}$ matrix with rows and columns indexed by the vertices and edges of $G$, respectively, such that $B_{i j}=1$ if the vertex $i$ is the head of the edge $j, B_{i j}=-1$ if the vertex $i$ is the tail of the edge $j$, and 0 otherwise. The Laplacian matrix is given by $L=B B^{T}=\Delta-A[8]$.

The cycle space of a graph will be useful in the sequel. If the graph $G$ contains cycles, then its cycle space is the subspace spanned by vectors representing cycles in $G$ [9]. The edges of each cycle in $G$ have a direction, where each edge is directed towards its successor according to the cyclic order. A cycle $C$ is represented by a vector $v_{C}$ with number of elements equal to the number of edges $M$ of the graph. For each edge, the corresponding element of $v_{C}$ is equal to 1 if the direction of the edge with respect to $C$ coincides with the orientation assigned to the graph for defining the incidence matrix $B$, and -1 , if the direction with respect to $C$ is opposite to the orientation. The elements corresponding to edges not in $C$ are zero.

In this paper we use a sign definiteness property of the matrix $B^{T} B$. While $L$ is always positive semidefinite, the matrix $B^{T} B$ can be either positive semidefinite or positive definite. The next lemma states that in the case of a tree graph, the matrix $B^{T} B$ is always positive definite:

Lemma 1: If $G$ is tree, then $B^{T} B$ is positive definite. Proof: For arbitrary $y \in \mathbb{R}^{M}$ we have $y^{T} B^{T} B y=|B y|^{2}$ and hence $y^{T} B^{T} B y>0$ if and only if $B y \neq 0$, i.e., the matrix $B$ has empty null space. For a connected graph, the cycle space of the graph coincides with the null space of $B$ (Lemma 3.2 in [9]). This corresponds to that for $G$, which has no cycles, zero is not an eigenvalue of $B$. This implies that $\lambda_{\min }\left(B^{T} B\right)>0$, i.e., that $B^{T} B$ is positive definite. $\diamond$

We note here that the matrix $B^{T} B$ is defined as the "Edge Laplacian" in [25] and its properties are used for providing another perspective to the agreement problem.

\section{CONTROL LAW AND STABILITY ANALYSIS}

Denote by $\beta_{i j}(q)=\left\|q_{i}-q_{j}\right\|^{2}$ the Euclidean distance of any pair of agents in the group. The class $\Gamma$ of formation potentials $\gamma \in \Gamma$ between agents $i$ and $j$ with $j \in N_{i}$ is defined to have the following properties:

1) $\gamma: \mathbb{R}^{+} \rightarrow \mathbb{R}^{+} \cup\{0\}$ is a function of the distance between $i$ and $j$, i.e., $\gamma=\gamma\left(\beta_{i j}\right)$,

2) $\gamma\left(\beta_{i j}\right)$ is continuously differentiable,

3) $\gamma\left(d_{i j}^{2}\right)=0$ and $\gamma\left(\beta_{i j}\right)>0$ for all $\beta_{i j} \neq d_{i j}^{2}$.

We also define

$$
\rho_{i j} \triangleq \frac{\partial \gamma\left(\beta_{i j}\right)}{\partial \beta_{i j}}
$$

Note that $\rho_{i j}=\rho_{j i}$, for all $i, j \in \mathcal{N}, i \neq j$. The proposed control law is

$$
u_{i}=-\sum_{j \in N_{i}} \frac{\partial \gamma\left(\beta_{i j}(q)\right)}{\partial q_{i}}=-\sum_{j \in N_{i}} 2 \rho_{i j}\left(q_{i}-q_{j}\right), i \in \mathcal{N}
$$

The set of controls (4) is written in stack vector form as

$$
u=-2\left(R \otimes I_{2}\right) q
$$


where $u=\left[u_{1}^{T}, \ldots, u_{N}^{T}\right]^{T}$ and the symmetric matrix $R$ is given by

$$
R_{i j}=\left\{\begin{array}{l}
-\rho_{i j}, j \in N_{i} \\
\sum_{j \in N_{i}} \rho_{i j}, i=j \\
0, j \notin N_{i}
\end{array}\right.
$$

We examine the stability of the closed loop system by using the candidate Lyapunov function

$$
V(q)=\sum_{i} \sum_{j \in N_{i}} \gamma\left(\beta_{i j}(q)\right)
$$

Its gradient can be computed as $\nabla V=4\left(R \otimes I_{2}\right) q$, so that its time-derivative is given by

$$
\dot{V}=-4\left\|\left(R \otimes I_{2}\right) q\right\|^{2} \leq 0
$$

We next show that the system reaches a static configuration:

Theorem 2: Assume that the system (1) evolves under the control law (4), and that the formation graph is connected. Then $u_{i}(t) \rightarrow 0$ as $t \rightarrow \infty$ for all $i \in \mathcal{N}$.

Proof: The level sets of $V$ are compact and invariant with respect to the relative positions of adjacent agents. Specifically, the set $\Omega_{c}=\{q: V(q) \leq c\}$ for $0<c<\infty$ is closed by continuity of $V$. From $V \leq c$ we have $\gamma\left(\beta_{i j}\right) \leq c$ for all $(i, j) \in E$. This implies that there is a $\xi$, where $0<\xi<\infty$, such that $\beta_{i j} \leq \xi$, by definition of the class $\Gamma$, and thus, $\left\|q_{i}-q_{j}\right\| \leq \sqrt{\xi}$ for all for all $(i, j) \in E$. Since the maximum length of the path between any two vertices of a connected graph is $N-1$, we have $0 \leq\left\|q_{i}-q_{j}\right\| \leq(N-1) \sqrt{\xi}$ for all $i, j \in \mathcal{N}$. Equation (5) and LaSalle's principle now guarantee that the system converges to the largest invariant subset of $S=\left\{q:\left(R(q) \otimes I_{2}\right) q=0\right\}$. Since $u=\dot{q}=-2\left(R \otimes I_{2}\right) q$, we have $u \rightarrow 0$ as $t \rightarrow \infty$ and the result follows. $\diamond$

We next provide a formation potential that guarantees formation stabilization for a class of formation graphs. In particular, we now consider the formation potential:

$$
\gamma\left(\beta_{i j}(q)\right)=\frac{\left(\beta_{i j}-d_{i j}^{2}\right)^{2}}{\beta_{i j}}
$$

Note that this potential satisfies all properties of the set $\Gamma$. Moreover, $\gamma\left(\beta_{i j}\right) \leq c \Rightarrow 0 \leq \frac{\left(\beta_{i j}-d_{i j}^{2}\right)^{2}}{\beta_{i j}} \leq c \Rightarrow$ $0 \leq\left(\beta_{i j}-d_{i j}^{2}\right)^{2} \leq c \beta_{i j} \Rightarrow \beta_{i j} \in[0, \xi]$ where $\xi=$ $\frac{1}{2}\left(2 d_{i j}^{2}+c+\sqrt{4 c d_{i j}^{2}+c^{2}}\right)$. For this case,

$$
\rho_{i j}=\frac{\partial \gamma\left(\beta_{i j}\right)}{\partial \beta_{i j}}=\frac{\beta_{i j}^{2}-d_{i j}^{4}}{\beta_{i j}^{2}}
$$

The next result involves the fact that with this choice of formation potential, communicating agents do not collide:

Lemma 3: Consider system (1) driven by the control (4) with $\gamma$ as in (6), and starting from a set of initial conditions $\mathcal{I}(q)=\left\{q \mid\left\|q_{i}-q_{j}\right\|>0, \forall(i, j) \in E\right\}$. Then $\mathcal{I}(q)$ is invariant for the trajectories of the closed loop system.

Proof: For every initial condition $q(0) \in \mathcal{I}(q)$, the time derivative of $V$ remains non-positive for all $t \geq 0$, by virtue of (5). Hence $V(q(t)) \leq V(q(0))<\infty$ for all $t \geq 0$. When $\left\|q_{i}-q_{j}\right\| \rightarrow 0$ for at least one pair of agents $i, j$, with $j \in N_{i}$, we have $V(q) \rightarrow \infty$, which is impossible. We conclude that $q(t) \in \mathcal{I}(q)$, for all $t \geq 0$. $\diamond$

Remark 1: Although collision avoidance is achieved between communicating agents, the issue of collision avoidance between non-communicating agents is not pursued here. This is an important requirement in realistic applications and thus should be considered in the model in future work.

The above result guarantees that $\beta_{i j}(t)>0$, i.e., $q_{i}(t) \neq$ $q_{j}(t)$, for all $t \geq 0$ and all $(i, j) \in E$. This will be used in the stability analysis of the closed-loop system.

We denote by $\bar{q}$ the $M$-dimensional stack vector of relative position differences of pairs of agents that form an edge in the formation graph, where $M$ is the number of edges, i.e, $M=|E|$ and $\bar{q}=\left[\bar{q}_{1}^{T}, \ldots, \bar{q}_{M}^{T}\right]^{T}$, where for an edge $e=(i, j) \in E$ we have $\bar{q}_{e}=q_{i}-q_{j}$.

With simple calculations, we can derive that $\dot{q}=$ $-2\left(R \otimes I_{2}\right) q$ is equivalent to

$$
\dot{\bar{q}}=-\left(B^{T} B W \otimes I_{2}\right) \bar{q}
$$

where the diagonal matrix $W$ is given by

$$
W=2 \cdot \operatorname{diag}\left\{\rho_{e}, e \in E\right\} \in \mathbb{R}^{M \times M}
$$

The convergence properties of the closed-loop system are now established in the following theorem:

Theorem 4: Assume that the system (1) evolves under the control law (4) with $\gamma$ as in (6), and that the formation graph is a tree. Further assume the desired formation is feasible, i.e., $\Phi \neq \emptyset$. Then the agents are driven to the desired formation, i.e., $\lim _{t \rightarrow \infty} q(t)=q^{*} \in \Phi$.

Proof: Since at steady state, $\dot{q}=u=-2\left(R \otimes I_{2}\right) q=0$, we also have $\dot{\bar{q}}_{e}=0$ for all $e \in E$ and thus $\dot{\bar{q}}=0$. Equation (8) yields $\left(B^{T} B W \otimes I_{2}\right) \bar{q}=0$.

By virtue of Lemma $1, B^{T} B$ is positive definite. Since $\left(B^{T} B W \otimes I_{2}\right) \bar{q}=0$, we then have

$$
\left(\left(B^{T} B\right)^{-1} \otimes I_{2}\right)\left(B^{T} B W \otimes I_{2}\right) \bar{q}=0,
$$

and thus $\left(W \otimes I_{2}\right) \bar{q}=0$. Since $W$ is diagonal, the last equation yields $\rho_{e} \bar{q}_{e}=0$ for all $e \in E$. Since $\rho_{e}$ is scalar this implies $\rho_{e}=0$ or $\bar{q}_{e}=0$. However, for all $e \in E$ we have $\bar{q}_{e}(t) \neq 0$ for all $t \geq 0$, due to Lemma 3 . We conclude that $\rho_{e}=0$ for all $e \in E$ at steady state and hence $\beta_{i j}=d_{i j}^{2}$, i.e, $\left\|q_{i}-q_{j}\right\|=d_{i j}$ for all $(i, j) \in E$, by virtue of (7). $\diamond$

Remark 2: Note that the potential $\gamma\left(\beta_{i j}\right)=\left(\beta_{i j}-\right.$ $\left.d_{i j}^{2}\right)^{2}$, as in [1], leads to the control law $u_{i}=$ $-\sum_{j \in N_{i}} 4\left(\beta_{i j}-d_{i j}^{2}\right)\left(q_{i}-q_{j}\right)$, which results in equilibria other than the desired ones (as also mentioned in [1]), even in the case of formation graphs that are trees. For example, some agents might get stuck to a common point. The result of Theorem 4 shows that the choice of $\gamma$ in the form (6) provides the desired convergence guarantees for tree graphs.

\section{A. Main result}

The proof of the previous theorem provides us the means to characterize the formation graphs for which a control law of the form (4) leads to the desired formation for any choice of potential function $\gamma \in \Gamma$. In particular, for any choice of 
$\gamma \in \Gamma$, the closed-system dynamics are given by $\dot{q}=u=$ $-2\left(R \otimes I_{2}\right) q$, or equivalently by

$$
\dot{\bar{q}}=-\left(B^{T} B W \otimes I_{2}\right) \bar{q}
$$

in the edge space. The analysis leading to Theorem 4 guarantees that $\left(B^{T} B W \otimes I_{2}\right) \bar{q}=0$ at steady state. By virtue of Lemma 1 , the matrix $B^{T} B$ is non-singular only when the formation graph contains no cycles. From the above discussion we derive the following result:

Theorem 5: Assume that the system (1) evolves under the control law (4) and that $\Phi$ is non-empty. Consider conditions (i) $u(q)=0$ only for $q \in \Phi$, and (ii) $\lim _{t \rightarrow \infty} q(t)=q^{*} \in \Phi$. Then there exists a formation potential $\gamma \in \Gamma$ such that (i),(ii) hold if and only if the formation graph is a tree.

Proof: The "if" part is shown in Theorem 4, with the choice of formation potential field (6).

For the "only if part", assume that the closed-loop system has reached a steady state at which $u=0$, which implies that $\left(B^{T} B W \otimes I_{2}\right) \bar{q}=0$. We will show that condition (i) cannot hold if $G$ is not a tree.

If the formation graph is not a tree, then the matrix $B^{T} B$ in singular and then the null space of $B$, and thus $B^{T} B$, is nonempty. In fact, in this case, using properties of Kronecker products [12], [2], we have

$$
\begin{aligned}
& \left(B^{T} B W \otimes I_{2}\right) \bar{q}=0 \Rightarrow\left(B^{T} B \otimes I_{2}\right)\left(W \otimes I_{2}\right) \bar{q}=0 \Rightarrow \\
& \left(\bar{q}\left(W \otimes I_{2}\right)\right)^{T}\left(B^{T} B \otimes I_{2}\right)\left(W \otimes I_{2}\right) \bar{q}=0 \Rightarrow \\
& \bar{q}^{T}\left(B W \otimes I_{2}\right)^{T}\left(B W \otimes I_{2}\right) \bar{q}=0 \Rightarrow
\end{aligned}
$$

so that $\left(B W \otimes I_{2}\right) \bar{q}=0$. Denoting by $\bar{x}, \bar{y}$ the stack vectors of the elements of $\bar{q}$ in the $x$ and $y$ coordinates, the last equation yields $B W \bar{x}=B W \bar{y}=0$, i.e., $W \bar{x}, W \bar{y}$ belong to the null space of $B$. Since the graph contains cycles, the null space of $B$ is non-empty. Thus we cannot reach the conclusion of the proof of Theorem 4 that $\left(W \otimes I_{2}\right) \bar{q}=0$. In fact, equations $B W \bar{x}=B W \bar{y}=0$ have an infinite number of solutions, since the matrix $B^{T} B$ is now singular.

Thus condition (i) cannot hold if $G$ is not a tree. We conclude that (i) and (ii) hold only if $G$ is a tree. $\diamond$

The last result states that if the formation graph contains cycles, then we can not design a control law of the form (4) that stabilizes the agents to the desired relative distances. On the other hand, the previous analysis provides the means to characterize the equilibria of the system for any graph, based on the null space of the matrix $B$.

Note that the null space of $B$ coincides with the cycle space of $G$. The latter is spanned by the vectors representing the cycles in the graph. Although the set of solutions of equation $\left(B W \otimes I_{2}\right) \bar{q}=0$ is infinite when the graph contains cycles, we can get a geometrical characterization of the resulting equilibria based on the form of the formation potential (6). Without loss of generality, we assume $d_{i j}=d$ for all $(i, j) \in E$ from this point until the end of this section.

The vectors $W \bar{x}, W \bar{y}$ belong to the cycle space of $G$. Consider the case when $G$ has $p$ cycles, denoted by $C_{i}, i=$ $1, \ldots, p$ that do not have a common edge, i.e., the graph has $p$ disjoint cycles. Then we can rearrange the edges so that the edges corresponding to each cycle are successive and the edges that do not belong to a cycle are at the end of the edge sequence. It is easy to verify then that the cycle space is spanned by the $p$ vectors of dimension $M=|E|$, one for each cycle, who have 0 entries at elements not corresponding to the cycle, and \pm 1 entries at elements corresponding to the cycle, depending on the agreement between edge and cycle orientation. Since the cycles are disjoint, the edges $e_{k}^{i} \in C_{i}, k=1, \ldots, m_{i}$ corresponding to the same cycle $C_{i}, i=1, \ldots, p$ reach a configuration where each of them satisfies $\left(\rho_{e_{k}^{i}} \otimes I_{2}\right) \bar{q}_{e_{k}^{i}}= \pm c_{i}$ for all $k=1, \ldots, m_{i}$, where $c_{i}=\left[c_{x i}^{T}, c_{y i}^{T}\right]^{T}$ is a constant vector. Denoting as $\bar{x}_{e_{k}^{i}}, \bar{y}_{e_{k}^{i}}$ the coefficients of $\bar{q}_{e_{k}^{i}}$, we have $\left(\rho_{e_{k}^{i}} \otimes I_{2}\right) \bar{q}_{e_{k}^{i}}= \pm c_{i} \Rightarrow$ $\left|\rho_{e_{k}^{i}} \bar{x}_{e_{k}^{i}}\right|=\left|c_{x i}\right|,\left|\rho_{e_{k}^{i}} \bar{y}_{e_{k}^{i}}\right|=\left|c_{y i}\right|$, for all $k=1, \ldots, m_{i}$. Thus, all elements of $W \bar{x}, W \bar{y}$ corresponding to edges of $C_{i}$ tend to the same absolute value at steady state. However, the specific expression of this common absolute value cannot be determined a priori. Unfortunately, this is the best that can be guaranteed for the steady state of a formation corresponding to a graph with cycles. Edges $e \in E$ that do not belong to a cycle satisfy the equation $\left(\rho_{e} \otimes I_{2}\right) \bar{q}_{e}=0$, and thus, the agent pairs that correspond to these edges attain the desired relative distance $d$ at steady state, according to the analysis at the end of the proof of Theorem 4. The previous discussion is summarized in the following corollary:

Corollary 6: Assume that the system (1) evolves under the control law (4) with formation potential given by (6). Further assume that the cycles contained in the formation graph are disjoint. Then all elements of $W \bar{x}, W \bar{y}$ corresponding to edges of $C_{i}$ tend to the same absolute value at steady state. Agent pairs that correspond to edges that do not belong to a cycle attain the desired relative distance $d$ at steady state.

Remark 3: The limitations of the last corollary should not be underestimated. The cycles of the graph need to be disjoint in order for the corollary to hold. Moreover, even in this case, the values of the edges' distances of each cycle cannot be predetermined and it certainly might be different than $d$, even if $\gamma \in \Gamma$ is given by (6).

Remark 4: As mentioned in the introduction, a formation is called rigid when its shape is preserved if the distances imposed by the formation graph are maintained. Therefore tree graphs are not rigid. By virtue of Theorem 5, rigid formations cannot be globally stabilized by control laws of the form (4). It is then evident that other control design logics are needed for stabilization of rigid based formations. This is the topic of ongoing research endeavors.

\section{APPLICATION TO FLOCKING MOTION}

In this section we consider double integrator agents (3). The objective is flocking in a pre-specified distance based formation. Thus, agents aim to converge to the desired formation described in the previous section and attain a common velocity. For this objective, we combine the results of [22],[18] with the results of the previous section. In particular, agents are equipped with a Laplacian control law in the velocity space, while they use the same distance based formation potential in the position space. 
The proposed control law for system (3) is given by

$$
v_{i}=-\sum_{j \in N_{i}} \frac{\partial \gamma\left(\beta_{i j}(q)\right)}{\partial q_{i}}-\sum_{j \in N_{i}}\left(u_{i}-u_{j}\right)
$$

The formation potentials $\gamma$ were defined in (6). The control law can be written in stack vector form as

$$
v=-2\left(R \otimes I_{2}\right) q-\left(L \otimes I_{2}\right) u
$$

where the matrix $R$ was defined in the previous section and $L$ is the Laplacian of the formation graph. Using now

$$
V_{0}=\frac{1}{2} \sum_{i} \sum_{j \in N_{i}} \gamma\left(\beta_{i j}\right)+\frac{1}{2} \sum_{i}\left\|u_{i}\right\|^{2}
$$

as a candidate Lyapunov function, we can compute

$$
\begin{aligned}
\dot{V}_{0} & =\left(\frac{1}{2} \nabla \sum_{i} \sum_{j \in N_{i}} \gamma\left(\beta_{i j}(q)\right)\right) \dot{q}+u^{T} \dot{u}= \\
& =u^{T}\left(2 R \otimes I_{2}\right) q-u^{T}\left(\left(2 R \otimes I_{2}\right) q+\left(L \otimes I_{2}\right) u\right) \\
& =-u^{T}\left(L \otimes I_{2}\right) u \leq 0
\end{aligned}
$$

The main result of this section is stated in the following theorem, whose proof uses similar arguments to the corresponding one in [22], but is included here for completeness.

Theorem 7: Assume that the system (3) evolves under the control law (4) with $\gamma \in \Gamma$ as in (6), and that the formation graph is a tree. Then the agents reach a configuration where they have a common velocity and $\lim _{t \rightarrow 0} q(t)=q^{*} \in \Phi$.

Proof: The level sets of $V_{0}$ are compact with respect to the agents relative positions and velocities. In particular, the set $\Omega_{c}^{0}=\left\{(q, u): V_{0}(q, u) \leq c\right\}$ for $c>0$ is closed by the continuity of $V_{0}$. By virtue of Theorem 2, we have $0 \leq$ $\left\|q_{i}-q_{j}\right\| \leq(N-1) \sqrt{\xi}$ for all $i, j \in \mathcal{N}$. Moreover $V_{0} \leq c$ implies that $\left\|u_{i}\right\| \leq \sqrt{2 c}$, for all $i \in \mathcal{N}$.

Equation $\dot{V}_{0}=-u^{T}\left(L \otimes I_{2}\right) u$ and LaSalle's Invariance Principle now imply that the agents converge to a configuration where $\left(L \otimes I_{2}\right) u=0$. Using the notation $u_{x}, u_{y}$ for the stack vectors of the elements of $u$ in the $x$ and $y$ coordinates, we have $L u_{x}=L u_{y}=0$, Since the formation graph is connected, this implies that both $u_{x}, u_{y}$ belong to $\operatorname{span}\{\overrightarrow{\mathbf{1}}\}$, implying that $u_{1}=\ldots=u_{N}=u^{*}$ at steady state. Moreover, $\dot{u}_{x}, \dot{u}_{y}$ belong to $\operatorname{span}\{\overrightarrow{\mathbf{1}}\}$. In $\left\{u \mid u_{1}=\ldots=u_{N}=u^{*}\right\}$, we have $\dot{u}=v=-2\left(R \otimes I_{2}\right) q=-\left(B W \otimes I_{2}\right) \bar{q}=$ $-\left(B \otimes I_{2}\right)\left(W \otimes I_{2}\right) \bar{q}$ which implies that both $\dot{u}_{x}$ and $\dot{u}_{y}$ belong to the range of the incidence matrix $B$, which is equal to $\operatorname{span}\{\overrightarrow{\mathbf{1}}\}^{\perp}$ for a connected graph [22]. Hence $\dot{u}_{x}, \dot{u}_{y} \in \operatorname{span}\{\overrightarrow{\mathbf{1}}\} \cap \operatorname{span}\{\overrightarrow{\mathbf{1}}\}^{\perp} \equiv 0$, and thus, $\dot{u}=v=0$ at steady state. This in turn implies that $\left(R \otimes I_{2}\right) q=0$ which implies that $\lim _{t \rightarrow 0} q(t)=q^{*} \in \Phi$, i.e., the agents reach the desired formation, by virtue of Theorem $4 . \diamond$

Note that the result of Theorem 5 can be applied to the case of the double integrator agents of this section as well.

\section{Simulations}

In this section we provide some simulation examples to support the derived results.

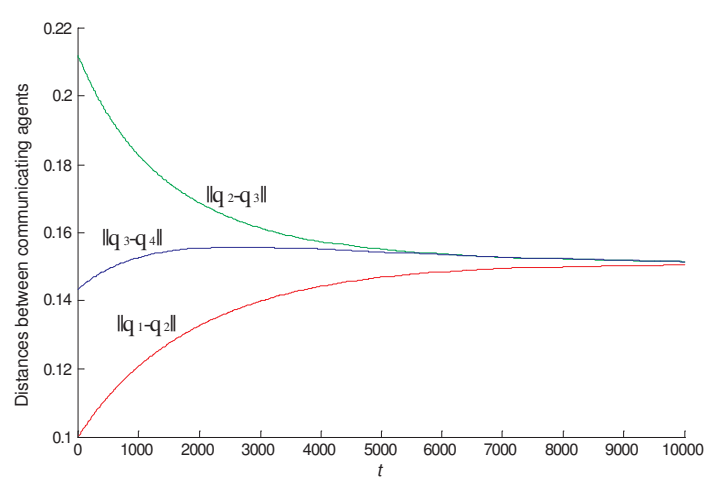

Fig. 1. Four single integrator agents are driven by the control (4) with $\gamma$ as in (6). The formation graph is a tree. Agents' relative distances are stabilized to the expected desired value $d=0.15$.

The first simulation involves four agents whose formation graph is a line graph, and hence a tree. In particular, we have $N_{1}=\{2\}, N_{2}=\{1,3\}, N_{3}=\{2,4\}$ and $N_{4}=\{3\}$. We have chosen the desired distance as $d=0.15$ for all edges in this example. Agents are driven by the control (4) with $\gamma$ as in (6). Figure 1 shows the plots of the distances $\left\|q_{1}-q_{2}\right\|,\left\|q_{2}-q_{3}\right\|$ and $\left\|q_{3}-q_{4}\right\|$, respectively. As witnessed in the figure, all distances between the communicating agents converge to the expected value $d=0.15$.

The second simulation is an example where the control law of the form (4) fails to stabilize a system of three agents to a triangular formation. The graph considered is a cycle graph and is complete, i.e., $N_{1}=\{2,3\}, N_{2}=\{1,3\}$, $N_{3}=\{2,3\}$. We also have $d_{12}^{2}=d_{13}^{2}=d_{23}^{2}=d^{2}=\sqrt{2}$ in this example and apply the potential (6) in the control (4). The agents start from initial positions $q_{1}(0)=[0,0]^{T}$, $q_{2}(0)=[-1,0]^{T}$ and $q_{3}(0)=[1,0]^{T}$. The evolution of the system is depicted in Figure 2, where the crosses represent the initial positions of the agents and their final locations are noted by a black circle. The system converges to an undesired configuration given by $q_{1}=[0,0]^{T}, q_{2}=[-0.6866,0]^{T}$ and $q_{3}=[0.6866,0]^{T}$. Simple calculations yield $u_{1}=u_{2}=$ $u_{3}=0$ at this configuration, so the system is at steady state. This is due to that the edge distances belong to the cycle space of the graph, i.e., we have $B\left(W \otimes I_{2}\right) \bar{q}=0$ and $\left(W \otimes I_{2}\right) \bar{q} \neq 0$ at steady state in this example.

By slightly altering the initial conditions of the previous example, the agents are driven to the desired triangular formation, as depicted in the simulation of Figure 3. The only difference is that we now have $q_{2}(0)=[-1,0.04]^{T}$. The system converges to the desired formation, even if the formation graph is a cycle. Thus, not all initial conditions are attracted to undesirable equilibria that belong to the cycle space of $G$ and not in $\Phi$, as encoded in Theorem 5. The characterization of the undesirable sets of initial conditions that are attractors to the cycle space of the graph $G$ is a topic of ongoing research. The use of Navigation Function theory [15] and Dual Lyapunov theory [4], [20] may provide the means for this research direction. 


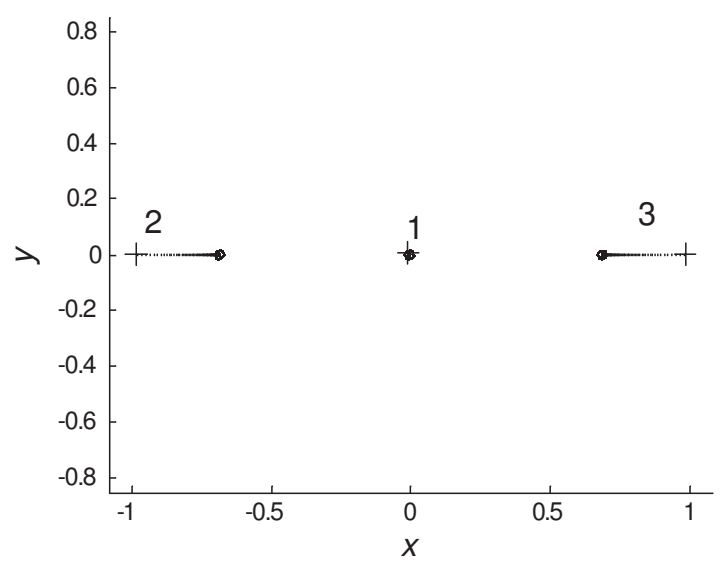

Fig. 2. Three agents with control law (4),(6) and a complete formation graph fail to reach the desired triangular formation. The resulting configuration belongs to the cycle space of the graph and does not coincide with a point in $\Phi$.

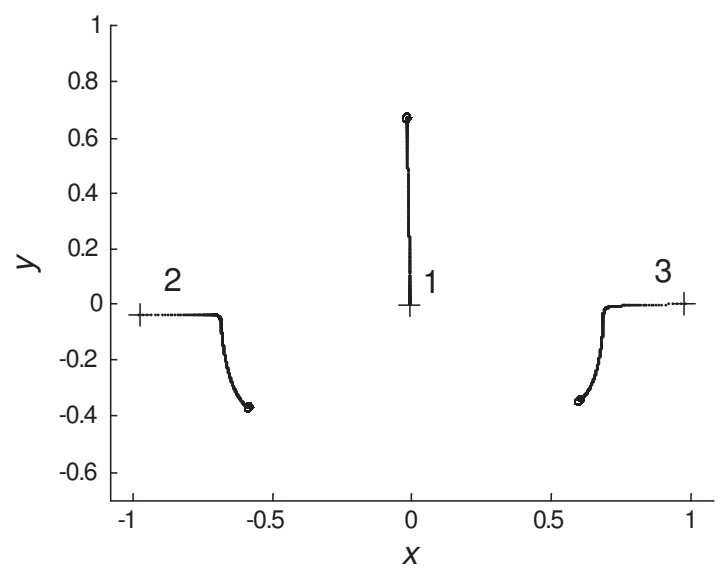

Fig. 3. By slightly altering the initial conditions of the previous example however, the agents are driven to the desired triangular formation.

\section{CONCLUSION}

We examined stability properties of distance-based formations. These are formations encoded by relative distances between pairs of agents that form an edge in the formation graph. A negative gradient control law was proposed and was shown to be provably correct when the formation graph is a tree. Moreover, it was shown that the tree structure is a necessary and sufficient condition for distance-based formation stabilization with negative gradient control laws. For graphs that contain cycles, a characterization of the resulting equilibria was given based on the properties of the cycle space of the graph. The results were also extended to the case of flocking motion for double integrator agents. Simulation examples supported the derived results.

Future research will involve extending the results to the case of directed graphs. We also aim to characterize the undesirable sets of initial conditions that are attractors to the cycle space of the formation graph.

\section{REFERENCES}

[1] J. Baillieul and A. Suri. Information patterns and hedging Brocketts theorem in controlling vehicle formations. 43rd IEEE Conf. Decision and Control, pages 556-563, 2004.

[2] J.W. Brewer. Kronecker products and matrix calculus in system theory. IEEE Transactions on Circuits and Systems, 25(9):772-781, 1978.

[3] J. P. Desai, J. Ostrowski, and V. Kumar. Controlling formations of multiple mobile robots. Proc. of IEEE Int. Conf. on Robotics and Automation, pages 2864-2869, 1998.

[4] D.V. Dimarogonas and K.H. Johansson. Analysis of robot navigation schemes using Rantzer's dual Lyapunov theorem. American Control Conference, pages 201-206, 2008.

[5] D.V. Dimarogonas and K.J. Kyriakopoulos. A connection between formation infeasibility and velocity alignment in kinematic multi-agent systems. Automatica, 2008. to appear.

[6] T. Eren, O.N. Belhumeur, W. Whiteley, B.D.O. Anderson, and A.S. Morse. A framework for maintaining formations based on rigidity. 15th IFAC World Congress, 2002.

[7] T. Eren, W. Whiteley, A.S. Morse, O.N. Belhumeur, and B.D.O. Anderson. Information structures to secure control of globally rigid formations. American Control Conference, pages 4945-4950, 2004.

[8] C. Godsil and G. Royle. Algebraic Graph Theory. Springer Graduate Texts in Mathematics \# 207, 2001

[9] S. Guattery and G.L. Miller. Graph embeddings and laplacian eigenvalues. SIAM Journ. Matrix Anal. Appl., 21(3):703-723, 2000.

[10] J.M. Hendrickx. Graph and Networks for the Analysis of Autonomous Agent Systems. PhD thesis, Faculté des sciences appliquées, École polytechinqe de Louvain, 2008.

[11] J.M. Hendrickx, B.D.O. Anderson, and V.D. Blondel. Rigidity and persistence of directed graphs. 44th IEEE Conf. Decision and Control, pages 2176-2181, 2005.

[12] R. A. Horn and C. R. Johnson. Matrix Analysis. Cambridge University Press, 1996.

[13] M. Ji and M. Egerstedt. Distributed coordination control of multiagent systems while preserving connectedness. IEEE Transactions on Robotics, 23(4):693-703, 2007.

[14] M.Mazo Jr., A.Speranzon, K. H. Johansson, and X.Hu. Multi-robot tracking of a moving object using directional sensors. 2004 IEEE International Conference on Robotics and Automation, 2004.

[15] D. E. Koditschek and E. Rimon. Robot navigation functions on manifolds with boundary. Advances Appl. Math., 11:412-442, 1990.

[16] Z. Lin, B. Francis, and M. Maggiore. Necessary and sufficient graphical conditions for formation control of unicycles. IEEE Transactions on Automatic Control, 50(1):121-127, 2005.

[17] A. Muhammad and M. Egerstedt. Connectivity graphs as models of local interactions. Journal of Applied Mathematics and Computation, 168(1):243-269, 2005.

[18] R. Olfati-Saber. Flocking for multi-agent dynamic systems: Algorithms and theory. IEEE Transactions on Automatic Control, 51(3):401-420, 2006.

[19] R. Olfati-Saber and R.M. Murray. Graph rigidity and distributed formation stabilization of multi-vehicle systems. 41st IEEE Conf. Decision and Control, 2002.

[20] A. Rantzer. A dual to Lyapunov's stability theorem. Systems and Control Letters, 42:161-168, 2001.

[21] A. Ryan, M. Zennaro, A. Howell, R. Sengupta, and K. J. Hendrick. An overview of emerging results in cooperative UAV control. 43rd IEEE Conf. Decision and Control, pages 602-607, 2004.

[22] H.G. Tanner, A. Jadbabaie, and G.J. Pappas. Flocking in fixed and switching networks. IEEE Transactions on Automatic Control, 52(5):863-868, 2007.

[23] H.G. Tanner and A. Kumar. Formation stabilization of multiple agents using decentralized navigation functions. Robotics: Science and Systems, 2005.

[24] C. Tomlin, G.J. Pappas, and S. Sastry. Conflict resolution for air traffic management: A study in multiagent hybrid systems. IEEE Transactions on Automatic Control, 43(4):509-521, 1998.

[25] D. Zelazo, A. Rahmani, and M. Mesbahi. Agreement via the edge laplacian. 46th IEEE Conference on Decision and Control, pages 2309-2314, 2007. 